Artigo Original

Original Article

Amanda Brait Zerbeto ${ }^{1}$

Regina Yu Shon Chun²

Descritores

Qualidade de vida

Cuidadores

Criança

Adolescente

Transtorno de linguagem

Gagueira

Fonoaudiologia

Keywords

Quality of life

Caregivers

Child

Adolescent

Language disorders

Stuttering

Speech-language and hearing sciences

Endereço para correspondência:

Amanda Brait Zerbeto

R. dos Carangueijos, 143, Perequê,

Ilhabela (SP), Brasil, CEP: 11630-000.

E-mail: amandabrat@gmail.com

Recebido em: 7/4/2012

Aceito em: 19/10/2012

\section{Qualidade de vida dos cuidadores de crianças e \\ adolescentes com alterações de fala e linguagem}

\section{Quality of life of caregivers of children and adolescents with speech and language disorders}

Objetivo: Investigar a qualidade de vida dos cuidadores de crianças ou adolescentes com alterações de fala e linguagem de acordo com a perspectiva deles. Métodos: Participaram dois grupos, totalizando 40 sujeitos. O Grupo 1 foi composto por 20 cuidadores de crianças ou adolescentes com alterações de fala e linguagem entre 4 e 17 anos, pareados por idade com o Grupo Controle ou 2, que incluiu 20 de crianças ou adolescentes sem queixas de fala e linguagem. Para a coleta de dados, foram aplicadas duas questões abertas e um instrumento da Organização Mundial de Saúde, traduzido e adaptado para o português - World Health Organization Quality of Life Scale (WHOQOL) Abreviado. Os resultados de tal instrumento foram submetidos à análise estatística, e as perguntas abertas estavam sendo analisadas qualitativamente. Resultados: Na distribuição das mudanças de fala e linguagem, havia: gagueira (35\%), alterações de linguagem oral sem (35\%) e com causas neurológicas (30\%). Na análise dos escores do WHOQOL-abreviado, encontraram-se diferenças da qualidade de vida nos domínios físico $(1,1 \%)$, psicológico $(0,5 \%)$ e relações sociais $(1,8 \%)$. O Grupo 1 apresentou qualidade de vida mais insatisfatória; e nas abertas, boa e razoável. Já o Grupo 2 mostrou ótima e boa. A rotina clínica e os filhos foram mencionados como fatores que dificultam o autocuidado no Grupo 1. Conclusões: O menor escore do Grupo 1 no WHOQOL-abreviado foi compatível com achados das questões abertas, mostrando que aspectos como rotina de atendimentos clínicos e dificuldades de compreensão influenciam a qualidade de vida dos cuidadores. Os resultados reafirmam a necessidade de que, além de cuidadores, sejam cuidados, entendendo-os como um grupo digno de ações de saúde direcionadas aos mesmos.

\begin{abstract}
Purpose: To investigate the quality of life of caregivers of children and adolescents presenting speech and language disorders from their own perspective. Methods: Two groups participated, adding up to 40 subjects. Group 1 was composed by 20 caregivers of 4 to 17 years-old children or adolescents with speech and language disorders, paired by age with the Control Group or 2 that included 20 caregivers of children or adolescents with no speech and language complaints. Data collection was done using: two open questions and the World Health Organization instrument, which was translated and adapted to Portuguese language - the World Health Organization Quality of Life Scale (WHOQOL-BREF). The results were submitted to statistical analysis and the open questions were qualitatively analyzed. Results: The language disorders distribution showed: stuttering (35\%), non-neurological (35\%), and neurological oral language disorders (30\%). In the analysis of the WHOQOLBREF scores, there were quality of life differences regarding the physical (1.1\%), psychological $(0.5 \%)$, and social relationships (1.8\%) domains. Group 1 presented the most dissatisfying quality of life. Concerning the open questions, it presented good and reasonable characteristics and Group 2, good and very good. The clinical routine and children were mentioned as factors that hamper self-care in Group 1. Conclusions: The lowest score of Group 1 in the WHOQOL-BREF was consistent with the open questions results, showing that aspects such as clinical attendance routine and comprehension difficulties influence caregivers' quality of life. The results corroborate that they should be assisted, since they are a group that deserves healthy actions directed to them.
\end{abstract}

Trabalho realizado no Curso de Fonoaudiologia, Universidade Estadual de Campinas - Unicamp-Campinas (SP), Brasil. (1) Programa de Pós-graduação (Mestrado) em Saúde, Interdisciplinaridade e Reabilitação, Universidade Estadual de Campinas - Unicamp - Campinas (SP), Brasil.

(2) Departamento de Fonoaudiologia, Universidade Estadual de Campinas - Unicamp - Campinas (SP), Brasil. Conflito de interesse: nada a declarar. 


\section{INTRODUÇÃO}

A qualidade de vida (QV) dos cuidadores de crianças e adolescentes com agravos fonoaudiológicos pode ter influência direta na participação e no envolvimento dos mesmos no acompanhamento terapêutico. Um membro na família que exija cuidados especiais pode também influenciar nos aspectos emocionais, ambientais e físicos dos demais, especialmente do principal responsável por esta criança ou adolescente ${ }^{(1)}$.

Segundo o World Health Organization Quality of Life Assessment (WHOQOL Group), QV é definida como "a percepção do indivíduo de sua posição na vida, no contexto da cultura e sistema de valores nos quais ele vive e em relação aos seus objetivos, expectativas, padrões e preocupações ${ }^{(2) !}$. Nessa perspectiva, esta abrange tanto as necessidades básicas e materiais (alimentação, moradia, trabalho, educação, saúde ou lazer), como as não materiais (felicidade, liberdade, satisfações pessoal e profissional, escolaridade e inserção social ${ }^{(3)}$. Ao considerar a abrangência do termo QV, é possível relacioná-lo diretamente à saúde.

Devido a sua grande extensão territorial e às diferenças regionais, o Brasil é conhecido pelas desigualdades sociais que também se refletem na saúde da população ${ }^{(4)}$. Um marco importante para o desenvolvimento da saúde no país foi a criação do Sistema Único de Saúde (SUS), que foi instituído pela Constituição de 1988 e garantiu que a saúde fosse considerada direito de todos e dever do Estado. A partir daí, ganharam destaque ações de promoção e prevenção de saúde, as quais anteriormente não eram o enfoque ${ }^{(4)}$.

O desenvolvimento do setor da saúde pode ser observado nos dados apresentados pelo Instituto Brasileiro de Geografia e Estatística (IBGE) no Censo de 2010, em que, dentre outras informações, constam o aumento da expectativa de vida e a diminuição da mortalidade infantil ${ }^{(5)}$. O investimento em pesquisas e equipamentos hospitalares, assim como o dever do Estado de garantir saúde a todos, são alguns dos fatores que influenciaram na diminuição da mortalidade infantil e na sobrevivência de crianças com necessidade de contínuos cuidados e serviços de reabilitação especializados ${ }^{(6)}$. Com a maior expectativa de vida, o número de cuidadores responsáveis por crianças também cresceu, sendo necessárias assim ações dirigidas não somente a elas, mas também aos seus responsáveis.

A condição de saúde do indivíduo tem influência sobre sua QV. Com o objetivo de estudar tal repercussão, várias pesquisas têm sido realizadas, e instrumentos foram elaborados e testados para avaliar a QV, não somente de pessoas com algum problema de saúde, mas também de seus cuidadores ${ }^{(7,8)}$.

Um dos instrumentos criados pela Organização Mundial de Saúde (OMS) com o intuito de avaliar a QV consiste no WHOQOL. Primeiramente, construiu-se uma versão estendida (WHOQOL-100) com cem questões, e a partir da necessidade de um instrumento mais conciso, foi idealizada uma versão abreviada deste, o WHOQOL-abreviado, traduzido e adaptado para o idioma português brasileiro ${ }^{(9)}$.

O questionário WHOQOL-abreviado é um dos objetos utilizados para avaliar a QV de cuidadores de pessoas com as mais diversas condições, porém ainda pouco utilizado na
Fonoaudiologia. Os estudos costumam abranger os sujeitos atendidos na clínica, e não seus cuidadores, como é o caso de publicações sobre gagueira ${ }^{(10)}$. Na revisão da literatura voltada aos cuidadores de crianças e adolescentes, foram encontrados trabalhos nas áreas de Síndrome de Down ${ }^{(11)}$, autismo ${ }^{(12,13)}$, afasia $^{(14)}$ e paralisia cerebral ${ }^{(15,16)}$. Um deles que comparou QV de pais de crianças autistas com aqueles com filhos sem alterações mostrou que a QV dos cuidadores de autistas estava mais afetada nos domínios físico e nas relações sociais, e que aquela dos responsáveis por pessoas que necessitam de atendimento especializado e maiores cuidados pode estar prejudicada ${ }^{(12)}$.

Cuidador é a pessoa mais próxima do sujeito, que passa maior parte do tempo com ele e tem papel ativo no tratamento fonoaudiológico ${ }^{(14)}$. Em diversas situações, é um membro da família do indivíduo que necessita de cuidados especiais. Em várias áreas da saúde, têm sido alvos de vários estudos. No entanto, no âmbito da Fonoaudiologia, há poucos estudos com este propósito. $\mathrm{O}$ interesse de tais trabalhos advém da necessidade de atenção à saúde dos cuidadores e da criação de novas políticas públicas para prestação da formação e apoio de que necessitam ${ }^{(17)}$.

$\mathrm{Na}$ formação do profissional da saúde deve estar presente a capacitação para o novo modelo de saúde que está em diferentes propostas governamentais no Brasil, como o Programa de Saúde da Família (PSF), com enfoque não somente na doença do indivíduo como também em todo contexto que o cerca, incluindo a família ${ }^{(18)}$. Neste sentido, há uma tendência à valorização da família e de todas as pessoas envolvidas no processo saúde/doença da pessoa, o que reitera a importância de estudos como aqui proposto.

Um dos conceitos advindos desta nova concepção de saúde é a Clínica Ampliada(19), que prevê um atendimento que: "visa ao sujeito e à doença, à família e ao contexto, tendo como objetivo produzir saúde e aumentar a autonomia do sujeito, da família e da comunidade. Utiliza como meios de trabalho: a integração da equipe multiprofissional, a adscrição de clientela e a construção de vínculo, a elaboração de projeto terapêutico conforme a vulnerabilidade de cada caso, e a ampliação dos recursos de intervenção sobre o processo saúde-doença ${ }^{(19) "}$.

Desta forma, deve ser considerado o contex to do sujeito e não unicamente sua doença. Para que a saúde e o bem-estar de todos os envolvidos no processo sejam garantidos, políticas de saúde nacionais e internacionais, como as da OMS, preconizam e ressaltam a importância da atenção integral do sujeito, o que também inclui a família e seus cuidadores. Nesta perspectiva, o sujeito e a família, compreendidos em seu ambiente, terão espaço para serem vistos e atendidos.

Considerando-se que ainda são escassas as investigações voltadas aos cuidadores de sujeitos com agravos fonoaudiológicos, a importância do autocuidado, além do papel decisivo dos mesmos nos processos de reabilitação e inserção social de crianças e adolescentes com alterações de fala e linguagem, pesquisas com essa temática assumem relevância.

Assim, este estudo teve como objetivo investigar a QV dos cuidadores de crianças e adolescentes com alterações de fala e linguagem, na perspectiva dos mesmos, com vistas a aprimorar o olhar sobre as formas de acolhê-los de modo integral na clínica fonoaudiológica. 


\section{MÉTODOS}

\section{Desenho metodológico e local da pesquisa}

Tratou-se de um estudo seccional realizado em um Curso de Fonoaudiologia da Universidade Estadual de Campinas (Unicamp). A pesquisa foi aprovada sob n ${ }^{\circ} 379 / 2010$ pelo Comitê de Ética em Pesquisa da Faculdade da Unicamp, nos termos da Resolução 196/96 do Conselho Nacional de Saúde (Conep).

\section{Constituição do corpus}

Participaram do estudo dois grupos de cuidadores, totalizando 40 sujeitos. O Grupo 1 foi composto por 20 de crianças ou adolescentes, entre 4 e 17 anos, com alterações de fala e linguagem, atendidos em uma Clínica-escola de Fonoaudiologia, pareados por idade com o Grupo Controle ou 2, composto por 20 cuidadores de crianças ou adolescentes sem queixas de fala e linguagem.

\section{Procedimentos para coleta de dados}

Os dados foram coletados por meio do WHOQOLabreviado e duas perguntas abertas: fale um pouco sobre sua QV e sobre a reação das pessoas quanto às dificuldades de linguagem de sua criança ou adolescente, sendo a última questão aplicada somente no Grupo 1. Informações referentes à identificação geral dos participantes (idade, renda ou profissão) foram coletadas por meio da consulta ao prontuário.

As questões abertas, respondidas de forma escrita pelos cuidadores, foram aplicadas anteriormente ao questionário WHOQOL-abreviado, de modo a evitar que suas respostas sofressem influência do questionário aplicado em seguida.

O WHOQOL-abreviado, em sua versão traduzida e adaptada para o português ${ }^{(9)}$, é composto por 26 questões. Destas, duas são relacionadas à avaliação da QV e as demais contemplam quatro domínios: físico (dor, energia, sono, mobilidade); psicológico (sentimentos, autoestima, concentração); relações sociais (relações pessoais, apoio social, atividade sexual) e ambiente (segurança, recursos financeiros, cuidados de saúde, lar, transporte).

Cada pergunta tem a opção de ser respondida em uma escala de um a cinco pontos, sendo que os valores um e dois equivalem a aspectos negativos de insatisfação com a questão avaliada, a pontuação três revela um aspecto intermediário de satisfação com o aspecto avaliado e os valores quatro e cinco correspondem ao aspecto positivo de satisfação com o domínio avaliado.

A aplicação ocorreu de duas formas: os cuidadores do Grupo 1 foram reunidos, em sua maioria em pequenos grupos, quando foram dadas as instruções para o preenchimento do questionário e todos responderam no mesmo momento às questões. $\mathrm{O}$ mesmo processo descrito foi aplicado no Grupo 2, sendo que a aplicação também ocorreu de forma individual em alguns casos.

\section{Forma de análise dos dados}

Os depoimentos escritos pelos participantes da pesquisa foram analisados qualitativamente. As respostas de ambos foram comparadas, verificando-se os aspectos de QV em cada um deles.

Após aplicação do WHOQOL-abreviado, realizou-se o cálculo dos domínios padronizados do questionário e da média dos quatro domínios (físico, psicológico, relações sociais e ambiente). Foi traçado um perfil da QV de cada grupo, os dados foram comparados e foi verificada a significância estatística entre seus dados por meio do Teste de Wilcoxon (Breslow).

\section{RESULTADOS}

Perfil sociodemográfico dos cuidadores e distribuição das alterações de fala e linguagem das crianças e adolescentes

O perfil dos sujeitos indicou características semelhantes em ambos os grupos, como demonstrado na Tabela 1.

Tabela 1. Perfil dos cuidadores quanto à idade e renda mensal

\begin{tabular}{ccc}
\hline Variáveis & Grupo 1 & Grupo 2 \\
\hline Média de idade & 40,1 anos & 36,2 anos \\
Média da renda mensal & $\mathrm{R} \$ 1616,15$ & $\mathrm{R} \$ 1990,75$ \\
\hline
\end{tabular}

A média de idade do Grupo 1 foi de 40.1 anos, com faixa etária variando entre 20 e 66. No Grupo 2, a mesmo variou de 19 a 60 anos.

Em relação ao estado civil, no Grupo 1, 65\% dos cuidadores eram casados, $15 \%$ eram solteiros, $15 \%$ divorciados e $5 \%$ viúvos. No Grupo 2, 60\% eram casados, $30 \%$ solteiros e $10 \%$ divorciados.

Quanto às profissões no Grupo 1, 11 realizavam funções em casa (como costureira e do lar) e 9 trabalhavam em ambiente externo ao da residência. No Grupo 2, 18 deles trabalhavam fora de casa e 2 exerciam suas funções na residência.

Em relação ao gênero das crianças e dos adolescentes, o Grupo 1 foi composto de oito crianças ou adolescentes do gênero feminino e 12 do masculino. O Grupo 2 incluía dez do gênero feminino e dez do masculino.

As alterações de fala e linguagem das crianças ou adolescentes do Grupo 1 abrangeram: gagueira (35\%), alterações de linguagem oral sem $(35 \%)$ e com causas neurológicas $(30 \%)$. A média de tempo do acompanhamento fonoaudiológico foi de dois anos.

\section{Resultados do WHOQOL-abreviado}

Nos resultados do WHOQOL-abreviado (Tabela 2) foram verificadas diferenças significativas na QV entre os Grupos 1 e 2 nos domínios físico $(\mathrm{p}=1,1)$, psicológico $(\mathrm{p}=0,5)$ e relações sociais $(p=1,8)$, sendo que o Grupo 1 teve a QV mais insatisfatória em todos. No ambiente, encontrou-se uma maior relação de igualdade entre os dois grupos.

$\mathrm{Na}$ análise da QV do Grupo 1, os resultados indicaram que cuidadores de crianças e adolescentes do gênero feminino tiveram suas vidas mais afetadas do que aqueles do masculino, especialmente nos domínios de relações sociais e psicológico (Tabela 3).

A análise estatística da comparação entre a QV e as diferentes alterações de fala e linguagem (gagueira, alterações de linguagem com e sem causas neurológicas) não pode ser realizada devido ao tamanho amostral. 
Tabela 2. Média dos escores dos domínios da qualidade de vida (WHOQOL-abreviado), teste de Wilcoxon para comparar médias entre grupos

\begin{tabular}{cccc}
\hline \multirow{2}{*}{ Domínio } & \multicolumn{2}{c}{ Grupos (média) } & \multirow{2}{*}{$\begin{array}{c}\text { Teste de } \\
\text { Wilcoxon }\end{array}$} \\
\cline { 2 - 3 } & $1(\mathrm{n}=20) \%$ & $2(\mathrm{n}=20) \%$ & Wín \\
\hline Físico & 66,43 & 78,39 & $1,1^{*}$ \\
Psicológico & 64,17 & 77,92 & $0,5^{\star \star}$ \\
Relações sociais & 61,25 & 77,08 & $1,8^{\star}$ \\
Ambiente & 62,34 & 65,63 & 37,7 \\
\hline
\end{tabular}

Valores significativos ( $p \leq 0,05) ;{ }^{*}$ significativo para o nível de $5 \%$; ${ }^{* *}$ significativo para o nível de $1 \%$

Tabela 3. Comparação da qualidade de vida de acordo com os gêneros das crianças e adolescentes do Grupo I, teste de Wilcoxon para comparação de médias entre grupos

\begin{tabular}{cccc}
\hline & \multicolumn{2}{c}{$\begin{array}{c}\text { Gênero das crianças } \\
\text { e adolescentes }\end{array}$} & $\begin{array}{c}\text { Teste } \\
\text { Domínios }\end{array}$ \\
\cline { 2 - 3 } & $\begin{array}{c}\text { Feminino } \\
(\mathrm{n}=8) \%\end{array}$ & $\begin{array}{c}\text { Masculino } \\
(\mathrm{n}=12) \%\end{array}$ & \\
\hline Eísico & 59,37 & 71,13 & 6,8 \\
Psicológico & 54,68 & 70,48 & $3,3^{*}$ \\
Relações sociais & 43,75 & 72,91 & $0,8^{* *}$ \\
Ambiente & 57,81 & 65,36 & 38,4 \\
\hline
\end{tabular}

Valores significativos $(p \leq 0,05) ;{ }^{*}$ significativo para o nível de $5 \%$; ** significativo para o nível de $1 \%$

\section{Depoimentos dos cuidadores sobre suas qualidades de vida e a linguagem dos filhos}

Nas questões abertas, os cuidadores de crianças ou adolescentes, quando solicitados a falarem acerca de suas QV, abordaram pontos semelhantes em ambos os grupos. Foram levantados aspectos respectivos à alimentação, à moradia, aos bens materiais (roupas, transporte), ao trabalho, às relações sociais, ao lazer, à atividade física, à saúde, à estrutura familiar, aos aspectos financeiros, à religião e à ausência de vícios como bebida e cigarro. Tais participantes relacionaram a satisfação ou insatisfação em relação a esses aspectos para caracterizar sua QV.

No Grupo 2, de cuidadores de crianças ou adolescentes sem queixas de linguagem, a QV foi considerada como boa ou ótima na maioria dos casos, sendo que o foco das respostas foram os próprios sujeitos. Estes relacionaram o fato de terem moradia, sendo ela própria ou alugada, alimentação disponível para família e saúde como principais itens responsáveis pela QV que apresentavam.

O trabalho foi tratado como uma atividade que proporciona uma renda maior para a família, porém também restringe o tempo que os cuidadores passam com as crianças ou adolescentes. Mesmo sendo relatado que não seja grande, o fato não foi abordado como sendo totalmente negativo, pois vários sujeitos relataram que os períodos em que ficam juntos são de qualidade, como ilustra o depoimento do sujeito 34 (mãe):

Conseguimos tomar café da manhã e jantar sempre juntos. Nosso ambiente familiar é muito harmonioso. Durante as noites conversamos sobre nosso dia (todos falam). Oriento as crianças nas tarefas de casa. Organizamos casa, jantar, mochilas, uniformes e rezamos antes de dormir.
A condição financeira foi colocada, pela maioria, como um dos fatores que restringe as possibilidades de lazer da família, um dos motivos pelo qual os cuidadores referiram que, apesar de estarem satisfeitos com sua QV, esta ainda poderia ser melhor.

As respostas do Grupo 1 mostram que, embora os temas que apareceram tenham sido semelhantes aos dos cuidadores do Grupo 2, foram abordados de forma diferenciada, sempre relacionando a QV individual às alterações das crianças e dos adolescentes. Por exemplo, na questão "Fale um pouco sobre sua qualidade de vida", na totalidade das respostas, aparece a rotina das crianças ou adolescentes como um assunto que influencia diretamente a rotina. Isso é negativo na maioria dos casos, uma vez que destinam o tempo que teriam para cuidarem de si, para as demandas de cuidados de seus filhos. Desta forma, as dificuldades das crianças e dos adolescentes afetam, além dos mesmos, a QV de seus cuidadores.

Os responsáveis por crianças e adolescentes com alterações de fala e linguagem do Grupo 1 classificaram sua QV como boa e razoável. Relataram que a rotina dos filhos interfere diretamente nas suas, pois seus compromissos, além de escola, envolvem atendimentos especializados com outros profissionais como fonoaudiólogo, fisioterapeuta, neurologista, terapeuta ocupacional, psicólogo, dentre outros. Foi relatado que o compromisso de levar as crianças ou adolescentes a estes atendimentos é o principal fator de falta de tempo para que cuidem de suas próprias necessidades, como ilustra o depoimento do Sujeito 3 (mãe):

Trabalho bastante! Filhas para escola, ballet, fono, fisioterapia, dentista, catequese; trabalho no comércio de manhã; todo trabalho doméstico referente a cinco pessoas; toda parte administrativa e financeira (pago contas etc.); dois sábados por mês faço trabalho voluntário na pastoral. Sou zeladora da capela peregrina; levo a mãe ao médico. Nossa chega, já estou cansada.

Aspectos financeiros foram citados por três sujeitos como um impedimento para a realização de atividades de agrado familiar e também como um possível fator limitante de um desenvolvimento mais rápido do filho, por não terem condições financeiras de proporcionarem mais atendimentos especializados.

Outro item abordado nas respostas do Grupo 1 foi a importância do apoio fornecido pelo companheiro conjugal, pela família e por amigos. Por outro lado, nos casos em que os sujeitos eram divorciados ou não recebiam apoio satisfatório do parceiro, uma queixa constante foi a sobrecarga acarretada pelo cuidado, principalmente das crianças menores, como mostra o relato de um pai:

Falta tempo e dinheiro para fazer o que eu gosto. Tenho dois filhos, tem que levar no médico, na fono, na Sobrapar, no dentista, na Santa Casa e na creche. Minha vida podia melhorar se a mãe me ajudasse a cuidar dele. 
Os resultados mostram que, nas respostas do Grupo 1, foi constantemente abordado o tema das crianças ou adolescentes pelos quais os sujeitos eram responsáveis. Pelo fato delas necessitarem de atendimento especializado, diferentemente daquelas sem alterações de fala e linguagem, os cuidadores necessitam dedicar um período mais longo para acompanharem seus filhos nesses compromissos, ocupando um tempo que poderia ser dedicado a si próprio além do cuidado de suas crianças ou adolescentes. Desta forma, houve queixas de cansaço, falta de tempo para se dedicar às necessidades individuais e sobrecarga de atividades, fatores que refletem na QV deles.

Em relação à segunda questão feita apenas aos cuidadores do Grupo 1, "Fale um pouco sobre a reação das pessoas diante das dificuldades de linguagem", os sujeitos referiram dificuldade de comunicação com a criança e o adolescente e reação negativa de pessoas que não os conhecem, em grande parte das respostas, além de reações negativas na adolescência, especialmente de pessoas da mesma idade. Além disso, foram mencionadas mudanças na reação da família e de conhecidos após acompanhamento fonoaudiológico, que passaram a compreender melhor a criança e ou adolescente e estimular suas potencialidades, conforme relatos.

Quanto às dificuldades de comunicação, os cuidadores de crianças e adolescentes com alterações de fala e linguagem relataram dificuldade das mesmas em se comunicarem com pessoas que não mantêm contato constante. Isso não foi relatado apenas com pessoas desconhecidas, como também com os próprios cuidadores, especialmente com crianças e adolescentes com linguagem oral restrita e/ou que utilizam gestos para se comunicar.

Em relação à reação de pessoas que não conhecem a criança ou adolescente, foram relatadas, pelos cuidadores, diferenças na reação das pessoas que conhecem e daquelas que não conhecem-na. Pessoas da família e conhecidos parecem entender melhor a situação, buscando compreender suas falas e seus gestos, conforme os depoimentos dos sujeitos. No entanto, quem não os conhecem, para os cuidadores, apresentam maior dificuldade em entender suas falas, pedindo para repetir ou requisitando auxílio de seus acompanhantes. Inicialmente, foram identificadas as dificuldades das crianças/adolescentes, sendo que, em várias situações, os sentimentos de tristeza e compaixão transpareceram em suas falas, como ilustra o depoimento do Sujeito 18 (madrasta). Tal cuidadora referiu que isso incomodava não só os pais, como a própria criança:

Quando não sabem do AVC, criticam por ela usar um babador, porque já está grandinha. Mas quando falo, ficam com dó e sinto que ela não gosta desta situação.

Entretanto, com maior tempo de convivência, como referiu uma parte dos sujeitos, estratégias são descobertas para melhorar a comunicação e a criança/adolescente passa a ser vista não só pelas suas dificuldades, como também por suas capacidades.

Quanto às mudanças na reação da família e de conhecidos após a terapia fonoaudiológica, os cuidadores do Grupo 1 relataram mudanças no olhar sob a criança ou adolescente, como por exemplo, passando a valorizar também suas capacidades e não só os "erros". Alguns deles relataram que, nos casos de gagueira, falas como "respira, fala mais devagar, não fica nervoso" não foram mais utilizadas, sendo incentivadas as falas das crianças/adolescentes, sem que fossem interrompidos durante seus discursos, como explicita o Sujeito 3 (mãe):

No início, minha filha sofreu bastante, pois pessoas até riam da sua falta de fluência. Amigos da escola a maltratavam, tirando sarro. Nós mesmos não gostávamos da sua dificuldade em se expressar, no começo mandávamos ela respirar direito, ter calma, falar pausadamente. Erro. Depois fomos orientados a pararmos com essa conduta. Que era um erro, estávamos apenas reforçando sua dificuldade. Agora ela está super fluente.

\section{DISCUSSÃO}

$\mathrm{Na}$ análise dos resultados referentes aos quatro domínios do WHOQOL-abreviado, foram constatadas diferenças significativas em três domínios: físico, psicológico e relações sociais, com escores inferiores no Grupo 1, o que demonstra uma QV menor no grupo de cuidadores de crianças e adolescentes com alterações de fala e linguagem.

No físico, os cuidadores do Grupo 1 obtiveram uma média de $66,43 \%$, considerada regular. Os aspectos mais prejudicados foram sono e energia necessária no dia-a-dia, resultados similares encontrados em cuidadores de crianças com diferentes quadros, como paralisia cerebral ${ }^{(15,16)}$ e síndrome de Down ${ }^{(11)}$.

Os achados do questionário WHOQOL-abreviado foram condizentes com as respostas obtidas nas questões abertas, nas quais os cuidadores relataram sobrecarga nas atividades cotidianas e falta de tempo para se dedicarem a si próprio.

Aspectos como autoestima, imagem corporal e aparência, sentimentos negativos e positivos, memória e concentração fizeram parte do psicológico. No presente estudo, este foi o domínio do questionário WHOQOL-abreviado em que a diferença entre os grupos foi mais significativa. Em um estudo realizado com 30 pais de crianças com síndrome de Down, em que o WHOQOL-abreviado foi utilizado para analisar a QV, os autores também encontraram que o mais prejudicado, nesses cuidadores, foi o psicológico ${ }^{(11)}$. Alguns aspectos que podem estar relacionados a esse baixo escore são as sobrecargas psicológica, financeira e do cuidado com a criança, as quais podem eventualmente gerar sentimentos de incerteza e ansiedade ${ }^{(20)}$.

Em relação ao domínio das relações Sociais (satisfação com as relações pessoais, vida sexual e apoio de cuidadores, amigos e colegas de trabalho), este mostrou-se como um aspecto negativo da QV do Grupo 1. Fatores como auxílio de uma rede de apoio social, identificação com um grupo, aceitação da própria aparência e atribuição de sentido à própria vida são importantes para evitar a sobrecarga mental e o estresse nas pessoas ${ }^{(11,21)}$. Considerando-se que o mesmo encontrou-se prejudicado nos cuidadores do presente estudo, é importante estar atento a tais aspectos e destacar a importância da família na vida das crianças/adolescentes atendidas na clínica fonoaudiológica. 
Os resultados do WHOQOL-abreviado, demonstrando menores escores nos domínios psicológico e físico dos cuidadores de crianças e adolescentes com alterações de fala e linguagem, foram similares a alguns trabalhos, porém voltados mais especificamente ao autismo. Um estudo, que comparou a QV de pais e cuidadores de crianças autistas com pais de outras sem alterações, por meio da aplicação do WHOQOL-abreviado, encontrou que os domínios com menores escores no grupo de pais de autistas foram o físico e de relações sociais ${ }^{(12)}$.

Evidencia-se, desta forma, que, em famílias de crianças com alterações no desenvolvimento e nas quais existem dificuldades de interação e linguagem, a QV de seus cuidadores pode encontrar-se afetada. Reafirmando-se que ao compreender que esta influencia a vida da criança com dificuldades de fala e linguagem, são necessárias ações e cuidados de saúde voltadas para esse grupo, como estabelece o SUS sobre o cuidado integral com o sujeito, incluindo consequentemente seus cuidadores ${ }^{(4)}$.

Nos estudos em que o domínio psicológico mostrou-se afetado $^{(11,22-24)}$, destacou-se a importância de acompanhamento especializado para os pais, especialmente no campo da Psicologia, para favorecer, inclusive, atitudes positivas com as crianças pelas quais eram responsáveis.

No presente estudo, o gênero das crianças mostrou-se como um fator influenciador da QV de seus cuidadores. Em sua análise no Grupo 1, os resultados indicaram que cuidadores de crianças e adolescentes do feminino tiveram suas vidas mais afetadas, principalmente nos domínios psicológico e relações sociais (Tabela 3). Uma das hipóteses que poderia justificar tais achados reside no fato de que as crianças com problemas mais graves, especialmente os de causas neurológicas e que envolvam limitações físicas e mentais, pertenciam ao gênero feminino.

Nas respostas do Grupo 1, os filhos foram mencionados em todos os depoimentos, o que não ocorreu no Grupo 2. Isso evidencia as diferenças de demanda em função das alterações de fala e linguagem das crianças e dos adolescentes, que implicam cuidados especiais e maior dedicação dos pais. As rotinas de atendimentos especializados e atividades dos filhos foram constantemente abordadas e, em alguns casos, apresentadas como justificativa para a falta de tempo do cuidador para atividades de lazer e autocuidado, que não envolvessem a participação de sua criança ou adolescente ${ }^{(22,24)}$.

Os cuidadores do Grupo 1 citaram os filhos, relatando o quão a rotina de atendimentos influencia em suas expectativas pessoais e QV, resultados similares foram encontrados em cuidadores de crianças com diagnósticos que podem ter influência na fala e linguagem ${ }^{(11)}$. Com os objetivos de verificar os suportes sociais formal e informal recebidos pelos pais e caracterizar a QV percebida por eles, foi avaliada a percepção de 150 cuidadores por meio do WHOQOL-abreviado e de um questionário específico referente ao suporte social. Em relação ao formal recebido, $70 \%$ das crianças realizavam acompanhamento, ao menos com três profissionais especializados, situação que demonstra a disponibilidade que o cuidador necessita ter para acompanhá-las. Tempo este, em que a prioridade é a criança ou o adolescente, e não si próprio. Os pais relataram, em $68 \%$ dos casos, privação das situações de lazer e relações sociais, devido ao fato de apresentarem dificuldade em ter uma vida independente de seus filhos, sempre pensando em quem poderia cuidar da criança ${ }^{(25)}$.

Além disso, verifica-se que a condição da criança/adolescente influenciou na profissão que seus cuidadores exercem. Em relação às ocupações daqueles de crianças/adolescentes sem alterações de linguagem, estas, em sua maioria, são exercidas fora do ambiente domiciliar. No outro grupo, mais de 50\% dos sujeitos exerciam suas funções no domicílio, como dona de casa e costureira, o que mostra a influência do cuidar em diferentes esferas da vida, inclusive na possibilidade de escolha da atividade profissional.

O cansaço e a falta de tempo para si foram aspectos abordados pelos cuidadores de crianças/adolescentes com alterações de fala e linguagem ${ }^{(11,16)}$, porém tal fato foi pouco abordado no Grupo 2. Embora a falta de tempo tenha aparecido em algumas respostas do Grupo 2, a principal causa citada foi o excesso de trabalho e não o fato de terem que acompanhar as crianças e adolescentes em atendimentos e em outros compromissos, como ocorreu no Grupo 1.

Quanto à reação das pessoas diante das dificuldades de fala e linguagem da criança/adolescente, uma das questões mais abordadas foi a complexidade de comunicação com pessoas com pouco contato. Isso é encontrado em diversas patologias que podem interferir, especialmente, na eficácia da linguagem oral, como nos casos de Síndrome de Down ${ }^{(26)}$ e paralisia cerebral ${ }^{(27)}$. Por não conhecerem a criança/adolescente e, muitas vezes, não entenderem como ocorre a comunicação, desconhecidos apresentam dificuldade de compreender o que a criança/adolescente quer falar. Pela característica dialógica da linguagem ${ }^{(28)}$, pode haver dificuldade de se manter um diálogo quando uma parte não compreende a outra. Sendo assim, a conversa, muitas vezes, não tem continuidade.

Isso também pode acontecer com os cuidadores, especialmente no caso de crianças/adolescentes que utilizam gestos para se comunicar ou com linguagem oral reduzida ${ }^{(29)}$. Mesmo que ocorra com menor frequência, é importante sinalizar a dificuldade de comunicação no âmbito familiar, em função de sua repercussão na dinâmica cotidiana e influência na QV dos moradores da casa, os quais, diversas vezes, não compreendem os desejos e as necessidades das crianças/adolescentes e podem se sentir impotentes diante de tal situação.

As reações negativas relatadas, especialmente entre os adolescentes, podem levar ao isolamento social, levando-os a permanecer mais tempo em casa e/ou restringindo sua participação em atividades sociais. Além disso, podem haver momentos em que os cuidadores deixarão seus compromissos sociais para estar com seus filhos e, deste modo, a dificuldade desses também repercutirá em sua QV.

Estudos sobre a família atual e as possíveis repercussões que a presença de uma criança com deficiência na família pode acarretar apontam a importância do conhecimento das condições ambientais e do contexto em que vivem ${ }^{(30)}$. Discute-se a necessidade do profissional não conhecer unicamente a criança como também as diferentes esferas que a permeia, com ênfase em seus pais e cuidadores. Reiteram, deste modo, que os cuidadores desejam e merecem ser tratados com sensibilidade pelos profissionais que atendem seus filhos, sendo vistos 
como parceiros ativos no processo terapêutico e pessoas que também necessitam de cuidados ${ }^{(13)}$.

No presente estudo, o menor escore destes cuidadores no WHOQOL-abreviado foi compatível com as respostas apresentadas nas questões abertas, em que aspectos como rotina de saúde repercutiram, nem sempre de maneira positiva, na QV dos cuidadores. Os resultados demonstram, assim, a necessidade de ações de saúde direcionadas aos mesmos, que também necessitam ser vistos e considerados em sua integralidade.

\section{CONCLUSÕES}

No Grupo 2, dos cuidadores de crianças e adolescentes sem alterações de fala e linguagem, a QV foi considerada como boa ou ótima na maioria dos casos. No Grupo 1, com alterações de fala e linguagem, a QV foi caracterizada como boa ou regular, demonstrando as diferentes condições entre ambos em função das alterações de fala e linguagem de um deles.

O menor escore do Grupo 1 no WHOQOL-abreviado foi compatível com as respostas apresentadas nas questões abertas, mostrando que a rotina dos atendimentos de saúde, as dificuldades de compreensão, a reação das pessoas diante das dificuldades das crianças e adolescentes são aspectos que influenciam a QV dos cuidadores.

Diante dos resultados encontrados no WHOQOL-abreviado e da diferença relatada acerca da QV entre os grupos, é necessário reafirmar a necessidade de que estas pessoas, além de cuidar, também sejam cuidados, olhados como sujeitos e não somente como responsáveis pelas crianças e adolescentes com alterações em algum aspecto do desenvolvimento.

Diante das atuais políticas nacionais e mundiais de saúde em que aspectos como humanização, acolhimento e propostas como a Clínica Ampliada preconizam a atenção integral ao sujeito, o que implica incluir a família e outros cuidadores, os achados do presente estudo reiteram a necessidade e a importância de um olhar especial dirigidos aos cuidadores das crianças/adolescentes atendidos na clínica fonoaudiológica. Portanto, esta abordagem certamente terá repercussões no desenvolvimento e na $\mathrm{QV}$ de todos os atores sociais envolvidos, sejam eles crianças, adolescentes ou cuidadores.

* ABZ realizou a pesquisa como Trabalho de Conclusão de Curso sob orientação da RYSC, sendo que ambas participaram da construção do artigo.

\section{REFERÊNCIAS}

1. Greenberg JS, Seltzer MM, Krauss MW, Chou RJ, Hong J. The effect of quality of the relationship between mothers and adult children with schizophrenia, autism, or Down syndrome on maternal well-being: the mediating role of optimism. Am J Orthopsychiatry. 2004;74(1):14-25.

2. The WHOQOL GROUP. Development of the WHOQOL: Rationale and Current Status. Int J Mental Health. 1994;23(3):24-56.

3. Minayo MCS, Hartz ZMA, Buss PM. Qualidade de vida e saúde: um debate necessário. Cienc Saúde Coletiva. 2000;5(1):7-18.

4. Paim J, Travassos C, Almeida C, Bahia L, Macinko J. The Brazilian health system: history, advances, and challenges. Lancet. 2011;377(9779):1778-97.
5. Brasil. Instituto Brasileiro de Geografia e Estatística [Internet]. Censo 2010. [cited 2011 Nov 30]. Available from: http://www.ibge.gov.br.

6. Santos HG, Andrade SM, Birolim MM, Carvalho WO, Silva AMR. Mortalidade infantil no Brasil: uma revisão de literatura antes e após a implantação do Sistema Único de Saúde. Pediatria (São Paulo). 2010;32(2):131-43.

7. Wayne DO, Krishnagiri S. Parent's leisure: the impact of raising a child with Down syndrome. Occup Ther Int. 2005;12(3):180-94.

8. Stoneman Z. Examining the Down syndrome advantage: mothers and fathers of young children with disabilities. J Intellect Disabil Res. 2007;51(Pt 12):1006-17.

9. Fleck MPA, Louzada S, Xavier M, Chachamovich E, Vieira G, Santos L, et al. Aplicação da versão em português do instrumento abreviado de avaliação da qualidade de vida “WHOQOL-bref”. Rev Saúde Pública. 2000;34(2):178-83.

10. Craig A, Blumgart $E$, Tran $Y$. The impact of stuttering on the quality of life in adults who stutter. J Fluency Disord. 2009;34(2):61-71.

11. Buzzato LL, Beresin R. Qualidade de vida dos pais de crianças portadoras da síndrome de Down. Einstein. 2008;6(2):175-81.

12. Fernandes FDM. Sugestões de procedimentos terapêuticos de linguagem em distúrbios do espectro autístico. In: Limongi SCO (org). Fonoaudiologia: informação para formação. Rio de Janeiro: Guanabara Koogan; 2003. p. 55-65.

13. Brobst JB, Clopton JR, Hendrick SS. Parenting children with autism spectrum disorders. Focus Autism Other Dev Disabl. 2009;24(1):38-49.

14. Panhoca I, Rodrigues AN. Avaliação da qualidade de vida de cuidadores de afásicos. Rev Soc Bras Fonoaudiol. 2009;14(3):394-401.

15. Carvalho JTM, Rodrigues NM, Silva LVC, Oliveira DA. Qualidade de vida das mães de crianças e adolescentes com paralisia cerebral. Fisioter Mov. 2010;23(3):389-97.

16. Prudente COM, Barbosa MA, Porto CC. Relation between quality of life of mothers of children with cerebral palsy and the children's motor functioning, after ten months of rehabilitation. Rev Latino-Am Enfermagem. 2010;18(2):149-55.

17. Bookman A, Harrington M. Family caregivers: a shadow workforce in the geriatric health care system? J Health Polit Policy Law. 2007;32(6):1005-41.

18. Gabardo RM, Junges JR, Selli L. Arranjos familiares e implicações à saúde na visão dos profissionais do Programa Saúde da Família. Rev Saúde Pública. 2009;43(1):91-7.

19. Brasil. Ministério da Saúde do Brasil. Secretaria de Atenção à Saúde. Núcleo Técnico da Política Nacional de Humanização. HumanizaSUS: documento base para gestores e trabalhadores do SUS. 3a ed. Brasília: Editora do Ministério da Saúde; 2006.

20. Sloper P, Knussen C, Turner S, Cunningham C. Factors related to stress and satisfaction with life in families of children with Down's syndrome. J Child Psychol Psychiatry. 1991;32(4):655-76.

21. Shu BC. Quality of life of family caregivers of children with autism: The mother's perspective. Autism. 2009;13(1):81-91.

22. Schieve LA, Stephen JB, Catherine RSN, Coleen B. The relationship between autism and pareting stress. Pediatrics. 2007;119(Suppl 1):114-21.

23. Eker L, Tüzün EH. An evaluation of quality of life of mothers of children with cerebral palsy. Disabil Rehabil. 2004;26(23):1354-9.

24. Tuna H, Ünalan H, Tuna F, Kokino S. Quality of life of primary of children with cerebral palsy: a controlled study with Short Form-36 questionnaire. Dev Med Child Neurol. 2004;46(9):646-8

25. Barbosa MRP. Suporte Social e qualidade de vida em familiares de crianças do espectro autista [dissertação de mestrado]. São Paulo (SP): Faculdade de Medicina da Universidade de São Paulo; 2010. 93 p.

26. Flabiano-Almeida FC, Limongi SCO. O papel dos gestos no desenvolvimento da linguagem oral de crianças com desenvolvimento típico e crianças com síndrome de Down. Rev Soc Bras Fonoaudiol. 2010;15(3):458-64.

27. Chun RYS. O desenvolvimento da comunicação não verbal através dos símbolos Bliss em indivíduo não falante portador de paralisia cerebral. Distúrb Comun. 1991;4(2):121-36.

28. Bakhtin M. Estética da Criação Verbal. 4 ed. São Paulo: Martins Fontes; 2003.

29. Sprovieri MHS, Assumpção Jr FB. Dinâmica familiar de crianças autistas. Arqu Neuro-psiquiatr. 2001;59(2-A):230-7.

30. Fiamenghi Jr GAJ, Messa AA. Pais, filhos e deficiência: estudos sobre as relações familiares. Psicol Cienc Prof. 2007;27(2):236-45. 